\title{
Occupation Anarchique Du Domaine Public Dans La Commune De Yopougon A Abidjan : Une Complicite Des Autorites Locales?
}

\author{
Coulibaly Yaya \\ Kra Kouadio Eugène \\ Coulibaly Amadou \\ Enseignant-chercheur, Laresp-IGT, \\ Université Félix Houphouët Boigny-Abidjan, Côte d'Ivoire
}

Doi: 10.19044/esj.2017.v13n32p248 URL:http://dx.doi.org/10.19044/esj.2017.v13n32p248

\begin{abstract}
Long perceived as an institutional response to the problem of local development, the decentralization adopted and introduced in the 1980s favored the creation of numerous communes in Côte d'Ivoire. These local and regional authorities, which in principle have legal personality and financial autonomy, are struggling to satisfy the expectations of politicians and populations. Consequently, the consequences are felt in space and we are witnessing an anarchic and illegal occupation of public servitudes as is the case in the commune of Yopougon in Abidjan. The present study is thus a contribution to the lasting resolution of the problem of the anarchic occupation of the public domain for the preservation of the urban landscape of municipalities. The main objective is to show the impact on the urban landscape of the use of the improvement of municipalities' own revenue. The methodology used to achieve this objective was based on the observation, inventory of the spaces documentary research, observation and maintenance with local and governmental authorities to obtain data on the mode of acquisition and enhancement of spaces. The results reveal that these areas are occupied with more or less the agreement of the various local and governmental authorities and their management remains problematic.
\end{abstract}

Keywords: Yopougon, public domain, occupation, anarchy

\section{Résumé}

Longtemps perçue comme une réponse institutionnelle au problème de développement local, la décentralisation adoptée et introduite dans les années 80 a favorisé la création de nombreuses communes en Côte d'Ivoire. Ces collectivités territoriales dotées en principe de la personnalité juridique et 
de l'autonomie financière peinent à donner satisfaction aux attentes du politique et des populations. Ainsi, les conséquences se font sentir sur l'espace et l'on assiste à une occupation anarchique et illégale des servitudes publiques comme c'est le cas dans la commune de Yopougon à Abidjan. La présente étude se veut donc une contribution à la résolution durable du problème de l'occupation anarchique du domaine public pour la préservation paysage urbain des communes. L'objectif principal visé est de montrer l'impact sur le paysage urbain du recours à l'amélioration des recettes propres aux communes. La méthodologie utilisée pour atteindre cet objectif s'est appuyée sur l'observation, l'inventaire des espaces la recherche documentaire, l'observation et l'entretien avec les autorités locales et gouvernementales pour avoir des données sur le mode d'acquisition et de mise en valeur des espaces. Les résultats révèlent que ces espaces sont occupés avec plus ou moins l'accord des différentes autorités tant locales que gouvernementales et leur gestion demeure toujours problématique.

Mots clés : Yopougon, domaine public, occupation, anarchie

\section{Introduction}

A la faveur de la décentralisation amorcée dans les années 1980 par les autorités ivoiriennes, les communes créées ont donné un nouveau souffle au processus de développement local. Cette politique de communalisation, axée sur l'instrument de l'Etat en tant que principal bailleur de fonds à ces collectivités (Etat providence), a très vite connu des difficultés liées à la crise économique des années 80 . En effet, la rareté des ressources et les difficultés de tensions ont impacté négativement les budgets tant au niveau du fonctionnement que de l'investissement de ces communes. Celles de la ville d'Abidjan, censées être nanties financièrement, n'ont pas échappé à cette conjoncture.

Dans la recherche de palliatifs pour soutenir le budget afin de réaliser les promesses des campagnes électorales, les élus locaux notamment les Maires et surtout ceux de la Ville d'Abidjan vont se lancer à la recherche de financement dans le but d'améliorer leurs ressources propres. Au-delà de ce recours à l'amélioration des capacités financières, se pose le problème de l'aménagement local basé sur une urbanisation organisée. Ce qui sous-entend que dans leurs quêtes de financement, les élus locaux doivent et devraient le faire en respectant les normes urbanistiques.

L'espace choisi pour la présente étude est la commune de Yopougon couvrant le côté Ouest de la ville d'Abidjan (Cf. figure 1). 


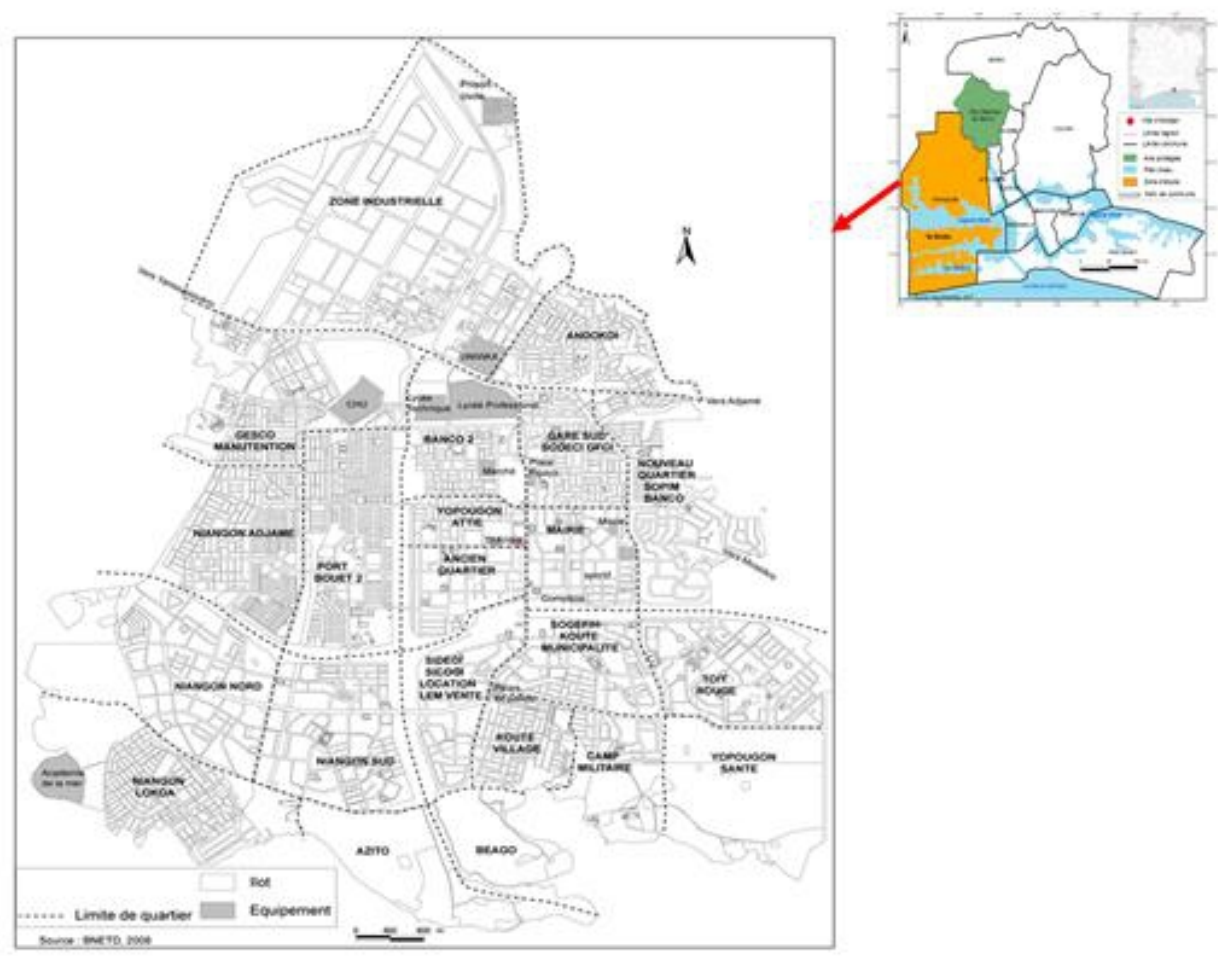

Figure 1 : Localisation et de présentation de la commune de Yopougon

C'est une commune populaire qui abrite plus du million d'habitants (1 071543 habitants selon l'INS, 2014) et s'étend sur une superficie de 156,03 km2 (DIABAGATE, 2013). A l'image des autres collectivités territoriales dotées en principe de la personnalité juridique et de l'autonomie financière, Yopougon peine à donner satisfaction aux attentes du politique et des populations. Ainsi, dans la commune on assiste à une forte pression sur l'espace ce qui entraîne une invasion et une occupation anarchique et illégale des servitudes publiques. De ce constat découle le problème de l'ampleur de l'occupation des servitudes publiques. Pour ce faire, l'objectif visé par l'étude est de montrer l'impact sur le paysage urbain de la complicité des autorités locales dans l'occupation anarchique des servitudes et domaines publics à travers une course effrénée de recherche de finances afin d'améliorer les ressources propres du budget. Pour atteindre cet objectif, nous analyserons d'une part la composition du budget de la commune de Yopougon et d'autre part, établirons l'état des lieux de l'occupation du domaine public.

\section{Methodologie}

La démarche méthodologique utilisée a consisté dans un premier temps à une phase observatoire. Ce qui nous a permis d'identifier et de 
classifier les espaces occupés. En effet, le domaine public en Côte d'Ivoire est constitué de biens immobiliers, de vastes étendues de terrains aux pourtours des aéroports, des ports, des gares ferronnières, le long des routes, des bordures de mer et de lagune avec de fortes potentialités économiques (ordonnance n²016-588 du 03 Août 2016).

Concernant la commune de Yopougon, l'étude s'est intéressée aux abords des voies principales, les bordures de lagune, les voies secondaires et les espaces publics sous les hautes tensions. Ces espaces sont l'objet de convoitise par les opérateurs économiques privés (petits comme grands commerces, marchés, transports) comme publics des secteurs du transport, de l'énergie, de la télécommunication. Ces logiques de gestion d'espace prévues par l'urbanisation combinées à celle d'une forte démographie dans la commune ont justifié notre choix.

Ensuite, nous avons eu recours à la recherche documentaire. Il s'agit des données des recettes qui ont permis d'apprécier le montant de la collecte de taxes lié à l'occupation de ces espaces et ce qu'elles représentent dans les recettes propres de la commune. Enfin, des entretiens avec trois autorités municipales (le directeur des affaires financières, le régisseur et le premier adjoint au Maire de la commune) ont permis de renforcer nos données.

\section{Resultats}

\section{Composition du budget de la commune de Yopougon}

La Commune de Yopougon fait partie des treize communes du District d'Abidjan qui ne bénéficient pas de la subvention de l'Etat à l'exception de la Commune d'Attécoubé (source enquête de terrain, 2016). Ainsi, les ressources mobilisables pour les actions de développement local et le budget reposent essentiellement sur les recettes variées et diverses. Principalement, elles sont composées de fiscalités locales, des revenus du patrimoine et des dotations de l'Etat.

Les recettes fiscales sont collectées par les services des impôts donc par l'Etat et dont une partie est rétrocédée à la commune. Cette part constitue 60 à $70 \%$ du budget de la collectivité. Et les recettes propres émanant des petites taxes, patentes et tickets journaliers collectés par les services de la régie de la commune représentent 30 à $40 \%$ du budget comme l'indique le tableau 1.

Tableau 1 : Etat des recettes de la commune de Yopougon

\begin{tabular}{|c|c|c|c|c|c|}
\hline Année & Budget & Recettes Fiscales & $\%$ & Recettes Propres & $\%$ \\
\hline 2013 & 5697207000 & 4949992000 & 86,88 & 747215000 & 13,11 \\
\hline 2014 & 4768797000 & 4068797000 & 85,32 & 700000000 & 14,67 \\
\hline 2015 & 5495000000 & 4420400000 & 80,44 & 1074600000 & 19,55 \\
\hline 2016 & 8497446000 & 4330250000 & 50,95 & 4167196000 & 49,04 \\
\hline
\end{tabular}

Source : Mairie de Yopougon (comptes Administratifs du Maire). 2013, 2014, 2014, 2015, 2016 
Ce budget se répartit entre l'investissement et le fonctionnement à des proportions variées selon les années (Cf. tableau 2).

Tableau 2 : Composition du Budget de 2013 à 2016 (en milliard)

\begin{tabular}{|c|c|c|c|c|c|c|c|c|}
\hline Titre du Budget & 2013 & $\%$ & 2014 & $\%$ & 2015 & $\%$ & 2016 & $\%$ \\
\hline Titre I & 5697 & 75,99 & 4768 & 81,94 & 5495000 & 75,62 & 8497 & 80,32 \\
Fonctionnement & 207 & & 797 & & & & 446 & \\
\hline Titre II & 1799 & 24 & $\begin{array}{c}1050 \\
857\end{array}$ & 18,05 & 1771537 & 24,38 & 2081 & 19,68 \\
Investissement & 215 & & & & 924 & \\
\hline TOTAL & 7496 & 100 & 5819 & 100 & 7266537 & 100 & $\begin{array}{c}10579 \\
370\end{array}$ & 100 \\
& 422 & & 654 & & & & \\
\hline
\end{tabular}

Source : Mairie Yopougon (Compte administratif du Maire), 2013, 2014, 2014, 2015, 2016

C'est donc au niveau des ressources propres représentant une proportion non négligeable (voir tableau 3) que sont collectées les recettes liées à l'occupation du domaine public (ODP). En réalité, la dénomination de l'ODP dans la délibération des taxes est «l'occupation sur permission administrative ».

Tableau 3 : Etat des recettes des ODP par rapport aux recettes

\begin{tabular}{|c|c|c|c|c|}
\hline ANNEE & 2013 & 2014 & 2015 & 2016 \\
\hline Recettes propres & 640133505 & 1042684720 & $\begin{array}{c}1181899 \\
030\end{array}$ & 1273415280 \\
\hline Recettes ODP & 49755453 & 74372750 & $\begin{array}{c}106384 \\
130\end{array}$ & 110701070 \\
\hline Part recettes ODP & $07,77 \%$ & $06,93 \%$ & $09 \%$ & $08,69 \%$ \\
\hline Recettes Fiscales & 3903155286 & 4422361434 & 4828507241 & 7683387121 \\
\hline
\end{tabular}

Sources : Mairie Yopougon Budget primitif 2013, 2014, 2014, 2015, 2016

\section{Etat d'occupation du domaine public dans la commune de Yopougon}

La Commune de Yopougon est la plus vaste et la plus peuplée de la Côte d'Ivoire (1 071543 habitants selon RGPH 2014 avec 156,03 km2 de surface) cependant, elle est une des rares communes ne disposant guère d'infrastructures socio-économiques (marchés) capables d'accueillir les activités commerciales. Les populations ont donc recours aux espaces capables de leur permettre de mener leurs activités. Ces espaces sont les abords des voies, des parkings, des lagunes, etc.... Ainsi, perçu naguère comme un espace hors du commerce, le domaine public est devenu aujourd'hui dans la commune de Yopougon, non seulement un objet de police administrative mais également une richesse collective que l'administration s'attache à gérer et exploiter le plus efficacement possible afin de le rentabiliser ce qui fait de sa protection une problématique (Cf. photos 1 et 2 ; figure 2 ). 


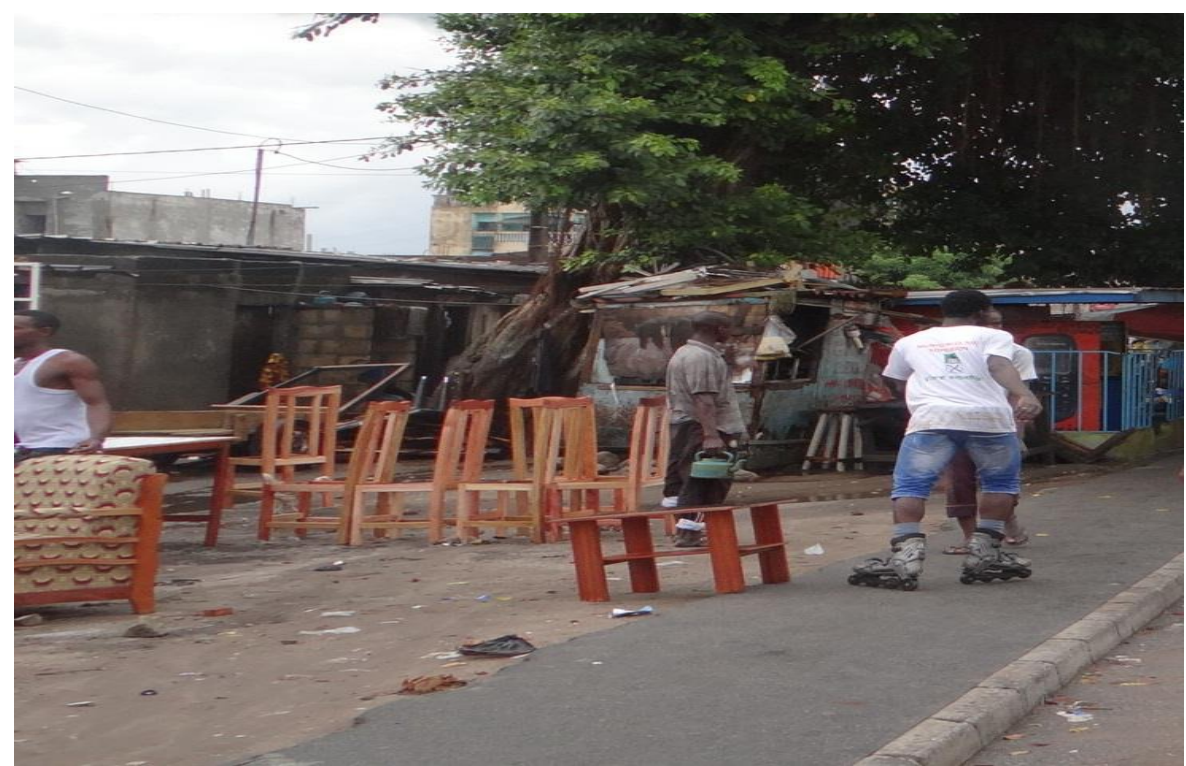

Photo 1 : Installation de menuiseries et d'autres types de commerces le long d'une voie (Voie principale $1^{\text {er }}$ pont, Bel air complexe sportif)

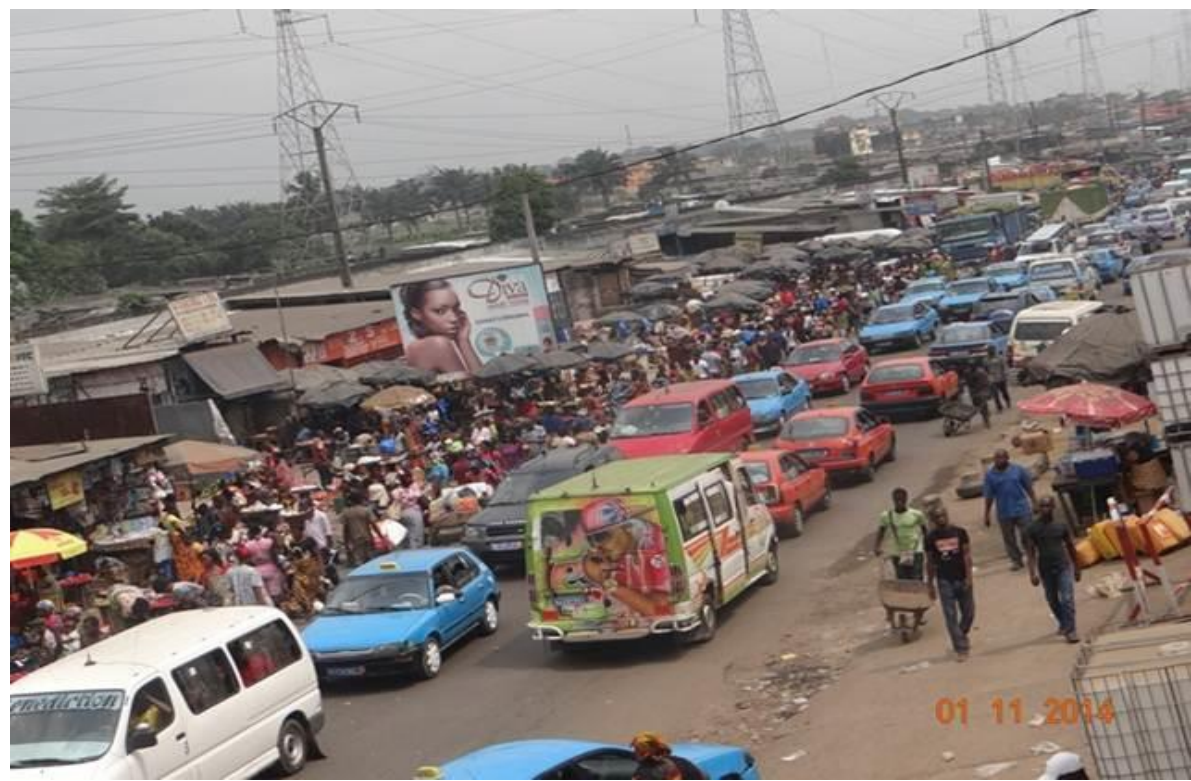

Photo 2 : Occupation de la voie publique par les commerçants (Quartier SICOGI) 


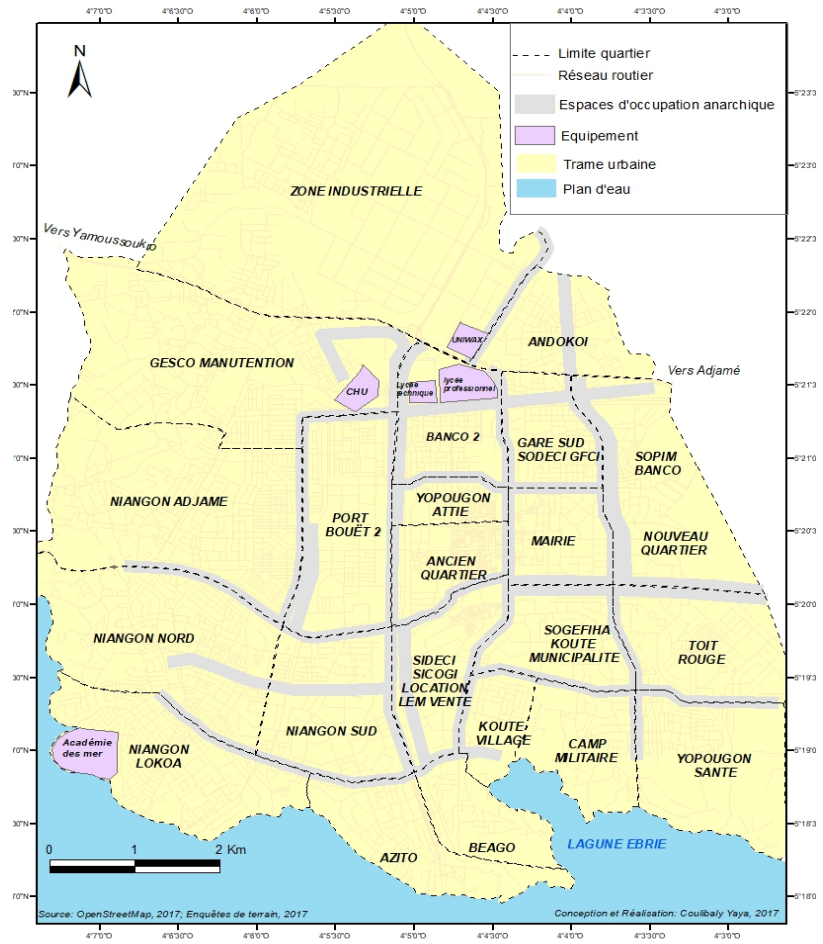

Figure 2 : Etat d'occupation du domaine Public

A ce jour, l'administration communale de Yopougon n'a délivré que 7402 autorisations administratives d'occupation temporaire (AOT) sur l'étendue du territoire communal et ce, pour une superficie occupée de 254 $800,594 \mathrm{~m} 2$ au titre des années 2015 et 2016. Les autorisations délivrées sont conformes aux dispositions de l'article 22, au titre d'autorisation d'occupation temporaire classique. Elles stipulent que les autorisations d'occupation précaires et révocables peuvent être délivrées par l'Etat, les collectivités territoriales, les établissements publics et les personnes morales de droit public ou privé ayant reçu mandat de la personne publique de gérer une partie de son domaine public. Cependant, cette autorisation est délivrée à l'issue d'une procédure. D'abord l'intéressé adresse un courrier au Maire, ensuite les services techniques, après investigations sur le terrain, donnent leur avis motivé et après validation le Maire prend un arrêté municipal autorisant le demandeur à occuper l'espace pour une année renouvelable. Celui-ci est donc autorisé à s'acquitter d'une redevance calculée au prorata du nombre de mètre carré à occuper. Ainsi, le droit d'installation sur le domaine public s'élève à 4000 FCFA (délibération du conseil municipal en date du 2 février 2015) par mètre carré et par année pour la première année (Année d'installation) et à $2500 \mathrm{Fcfa} / \mathrm{m}^{2}$ par année à partir de la deuxième année. 


\section{Discussion}

La politique fiscale instaurée par la communalisation pénalise les communes. En effet, depuis 1980, l'objectif principal visé par l'autorité en mettant en œuvre la décentralisation par la création des communes était que celles-ci soient capables d'impulser le développement local à partir d'une autonomie financière que l'autorité devrait lui concéder. Les Communes de l'intérieur du pays bénéficiant de la subvention de l'Etat et celles de la ville d'Abidjan (District d'Abidjan) jouissant d'une certaine autonomie apparente devraient être capables de s'auto-suffire en termes de recettes tant fiscales que recettes propres afin de soutenir leur développement local. La Commune de Yopougon, faisant partie de celles d'Abidjan, jusqu'en 2015 bénéficiait d'une proportion connu à l'avance des recettes fiscales collectées par les services des impôts de l'Etat et reverser au trésor. La part de la commune lui était donc reversée sous la base des taux de $40 \%$ de l'impôt foncier et $100 \%$ des vignettes auto.

Ces dispositions, connues d'avance, permettaient à la commune après information prise auprès des services des impôts délocalisés de projeter la part de la ristourne de la commune et par conséquent de préparer d'avance et à temps le budget primitif au plutard le premier trimestre de la nouvelle année. Cependant, depuis 2015 une nouvelle disposition, à travers une certaine solidarité fiscale, a été mise en place dans l'objectif de faire bénéficier aux autres communes du pays économiquement faibles des ristournes fiscales des grandes communes (dont celle de Yopougon). Le problème est que ni les services déconcentrés des impôts, ni même la commune ne maîtrise la base de calcul des ristournes allouées à la commune. La conséquence première d'une telle mesure est que la commune ne peut mettre en place son budget qu'avant le deuxième trimestre de la nouvelle année.

En outre, cette autonomie financière est biaisée par une forte dépendance des finances communales des dotations de l'Etat. Cette dépendance des collectivités territoriales vis-à-vis de l'Etat se mesure en termes de volume financier (MEYER, 2015).

Le budget de la Commune composé de deux types de recettes se subdivise entre l'investissement et le fonctionnement. L'investissement concerne les projets retenus dans le programme triennal et ne prend en compte que les travaux de construction d'infrastructures scolaires (...) de santé, achat de véhicules et réhabilitation d'engins marchands lourds (Plans triennaux de 2013 à 2016 de la mairie de Yopougon). Bien que la loi indique le caractère de l'autonomie financière reconnue aux collectivités territoriales, les communes produisent dans l'ensemble des ressources et dépendent pour l'essentiel des subventions et dotations de l'Etat pour les communes de l'intérieur du pays et des impôts d'Etat rétrocédés en ce qui concerne celles de la ville d'Abidjan dont Yopougon (KOFFI, 2012). Quant au fonctionnement, 
il concerne la bureautique, les salaires, les consommables (informatiques), les ordures ménagères. De ce fait, le budget des communes ne dépend que des actions de l'Etat à plus de $80 \%$. Les ressources propres que mobilisent les communes sont de l'ordre de 13 à $20 \%$. Elles sont composées des recettes des prestations de services. Ce sont entre autres les recettes des services généraux (timbres, vente d'autres documents), les recettes des services de collectivités (environnement, urbanisme, taxes d'enlèvement d'ordures ménagères, hygiène), les recettes des services sociaux culturels et de promotion humaine (taxes sur les actions culturelles, location de salle,...) et les recettes des services économiques (taxes sur le transport, la commission, le commerce, les marchés,...). Au titre des recettes propres, interviennent également le revenu du patrimoine et du portefeuille (le revenu du patrimoine immobilier, location de terrains, immeubles du domaine privé, occupation sur permission administrative ou ODP). L'asphyxie fiscale qu'exerce l'Etat sur les collectivités, au regard du volume financier collecté, contraint sans nul doute les communes à recourir à d'autres types de taxes afin de renforcer le budget dont la majeur partie (les $3 / 4$ ) est consacré au fonctionnement. Les taxes qui procurent les ressources propres sont diverses et ne sauraient être exhaustives. Car, toutes fois qu'une activité nait dans la commune et qu'elle est repérée par les services de la régie des taxes, une deliberation fixant le montant de la taxe liée à cette activité est prise en conseil municipal. Les différentes taxes occupent des proportions variées dans le budget. L'ODP au vu des chiffres du tableau 1 prend une part de 7 à $8 \%$ des recettes propres. Cette proportion non négligeable, ajoutée au fait que la commune doit multiplier ces ressources afin de les accroître pour soutenir son budget, amène les autorités à accorder les autorisations d'occupation temporaire du domaine et exploiter tous les espaces de la commune. La conséquence de cette course effrénée à la recherche de ressources est l'occupation anarchique des trottoirs, des espaces verts, des réserves administratives. En fait, certaines autorisations sont délivrées par le Ministère des Infrastructures Economiques pour occuper les abords des voies express et autoroutes. D'autres également sont délivrées par le Ministère de la Construction et le District d'Abidjan. C'est donc une foire à délivrance d'autorisation administrative à laquelle les populations assistent et dont la responsabilité n'est entièrement liée aux seuls responsables des communes. Il n'existe aucune plateforme entre les structures étatiques pour décider de la pertinence et de la nécessité de l'objet de la requête.

Pour ce faire, quelles stratégies pour conserver un environnement sain et sécurisé ? Il faut d'une part une autonomie financière. L'analyse établie à partir des enquêtes effectuées fait ressortir de façon évidente le problème des ressources financières qu'éprouvent les communes et plus particulièrement celle de Yopougon. La question fondamentale est quelle politique financière définir afin de permettre aux communes d'exercer leur politique de 
développement locale dans un environnement sain sans le détruire. Ainsi, la problématique d'une autonomie financière basée sur une viabilité financière des communes et leur capacité à mobiliser l'essentiel de leurs ressources budgétaires se posent désormais comme un des palliatifs nécessaires à un développement local et intégré des communes. Cette disposition prévue par la loi portant organisation des collectivités territoriales est battue en brèche dans la pratique rendant problématique le sens véritable de l'autonomie financière. A priori, les communes sont libres d'élaborer à travers leurs organes statutaires, d'adopter et d'exécuter leur budget, bien que ces budgets soient globalement tributaires des ristournes de l'Etat. Ainsi, l'autonomie financière ne doit pas être limitée uniquement à cette forme de liberté dont dispose les communes à élaborer, adopter et exécuter leur budget quelque soit la provenance des ressources. Elle doit plutôt se traduire en termes de capacité aux communes à disposer d'un budget élaboré essentiellement ou même principalement de ressources propres. L'Etat ivoirien doit donc responsabiliser davantage les communes en leur concédant une large autonomie financière à travers la collecte par elle-même d'une grande partie des ressources fiscales comme ressources propres. D'ailleurs, plusieurs associations de collectivités territoriales au plan international regroupées au sein des Cités et Gouvernements Locaux Unies (CGLU) reconnaissent que pour une meilleure efficacité des finances locales en matière de développement, l'autonomie financière devrait être accordée aux gouvernements locaux. Car, lorsque les finances reposent sur un grand pourcentage de transfert de l'Etat, cette situation engendre des rigidités et une dilution des responsabilités des Communes.

D'autre part, il faut concevoir une politique de transfert de compétences effective aux collectivités territoriales. En effet, pour conduire et soutenir la politique de développement des communes en Côte d'Ivoire, l'Etat central à mis en place un certain nombre de dispositifs tant législatifs que règlementaires dont la mise en œuvre souffre depuis environ des décennies (KOUASSI, 2013). Pour ce faire, un bilan des compétences transférées depuis l'avènement des communes en Côte d'Ivoire est nécessaire afin d'identifier les différents blocages à la mise en œuvre du processus et juger de la capacité des collectivités territoriales à prendre en charge ces différentes compétences. Ce qui permettra de redéfinir des décrets et leurs décrets d'application. En réalité, les ministères techniques ont une main mise sur les transferts des compétences aux collectivités en terme de finances si bien que celles-ci sont obligées pour un petit projet de construction (centre de santé, écoles) de le décliner en terme de programme triennal. Et parfois, l'Etat lui-même est constructeur en violation des règles que lui-même s'est fixé et qui stipulent que «dans le domaine de leurs compétences, les collectivités territoriales se substituent à l'Etat (article 36 de la loi n 2001-476 du 09 Août 2001). 
L'efficacité des collectivités passe obligatoirement par ce transfert réel des compétences tant au plan financier que humain. Cela suppose que les différents projets aient été financièrement évalués à travers les cartes sectorielles (cartes scolaires, sanitaires, etc....) et à charges aux ministères techniques de transférer les finances sur le compte de chaque collectivités.

\section{Conclusion}

La politique de communalisation amorcée en Côte d'Ivoire il y a des décennies peine à jouer pleinement son rôle de développement. Pour autant, son importance n'est plus à démontrer malgré le manque de moyen financier permettant à celle-ci de réaliser les projets adéquats pour le bien-être des populations. La commune de Yopougon n'échappe pas à cette réalité institutionnelle qui étouffe "énormément» le bon fonctionnement des collectivités territoriales. Le véritable souci est la question des transferts des compétences tant financières qu'humaines de l'Etat central aux communes. Et cette situation, qui relève d'une volonté politique, tarde à être appliquée depuis des décennies. Face à cette " asphyxie » financière, soutenue par une politique tutélaire encombrante plutôt que d'être encadreuse et conseillère, et face aux critiques des populations en l'endroit des élus (Maires) qui sont vue comme des incapables à booster le développement local, ceux-ci sont donc contraints de recourir à des méthodes leur permettant d'accroître leurs ressources financières propres afin de soutenir les budgets. Il s'agit pour la commune de Yopougon de tolérer les installations de commerce sur le domaine public certes mais qui lui permettent de soutenir les budgets à des propositions non négligeables. Cependant, elle laisse un paysage urbain indigent en proie à des installations anarchiques. Il convient donc de noter que si l'on veut des communes saines et propres, de mettre à disposition les moyens adéquats à ces collectivités qui passent nécessairement par le transfert effectif des compétences aussi bien financières que humaines.

\section{References:}

1. DIABAGATE A ., (2012), Importance numérique et répartition de la population dans l'agglomération d'Abidjan, Thèse unique de Doctorat en Géographie, IGT, Abidjan, 435p

2. MEYER G., (2015), Développement durable et finances locales, éditions l'Harmattan, Paris, 334p.

3. BONNET J., (2012), Aménagement et développement territorial, éditions ellipses, $362 \mathrm{p}$.

4. HAUHOUOT A., (2002), Développement, Aménagement et régionalisation en Côte d'Ivoire. Abidjan, EDUCI, Collection Essai, 309 p. 
5. ETAT GENERAUX DE LA DECENTRALISATION ET DU DEVELOPPEMENT LOCAL EN COTE D'IVOIRE : COMMISSION THEMATIQUE 3, (2007): Politique nationale de financement du développement local. Abidjan, $98 \mathrm{p}$.

6. BOUDON P., (1999), Langages singuliers et partagés de l'urbain, éditions l'Harmattan, $240 \mathrm{p}$.

7. KOUASSI Y.,(2013), Le processus de la décentralisation en Côte d'Ivoire, d'hier à aujourd'hui ;éditions Balafon, 340p. 